\title{
A Bernstein-Bézier Based Approach to Soft Tissue Simulation
}

\author{
S. H. Martin Roth ${ }^{\ddagger}$, Markus H. Gross ${ }^{\ddagger}$, Silvio Turello ${ }^{\ddagger}$, Friedrich R. Carls ${ }^{\dagger}$ \\ †Computer Science Department \\ ETH Zurich, Switzerland \\ e-mail: \{roth, grossm\}@inf.ethz.ch \\ http://www.inf.ethz.ch/department/IS/cg \\ †Department of Cranio-Maxillofacial Surgery \\ University Hospital, Zurich, Switzerland
}

\begin{abstract}
This paper discusses a Finite Element approach for volumetric soft tissue modeling in the context of facial surgery simulation. We elaborate on the underlying physics and address some computational aspects of the finite element discretization.

In contrast to existing approaches speed is not our first concern, but we strive for the highest possible accuracy of simulation. We therefore propose an extension of linear elasticity towards incompressibility and nonlinear material behavior, in order to describe the complex properties of human soft tissue more accurately. Furthermore, we incorporate higher order interpolation functions using a Bernstein-Bézier formulation, which has various advantageous properties, such as its integral polynomial form of arbitrary degree, efficient subdivision schemes, and suitability for geometric modeling and rendering. In addition, the use of tetrahedral Finite Elements does not put any restriction on the geometry of the simulated volumes.

Experimental results obtained from a synthetic block of soft tissue and from the Visible Human Data Set illus-
\end{abstract} trate the performance of the envisioned model.

Keywords: Physically Based Modeling, Finite Element Methods, Facial Surgery Simulation, Facial Modeling, Maxillofacial Surgery, Bernstein-Bézier, Soft Tissue.

\section{Introduction}

\subsection{Motivation}

Maxillofacial surgery and craniofacial surgery take care of a great variety of diseases of the whole face and skull, i.e. fractures, tumors, infections and malformations. Fractures of craniofacial bones have to be repositioned and fixed and a wide spectrum of facial and craniofacial malformations (e.g. figure 1) have to be treated. Some of the patients show only minor asymmetries, e.g. of the mandible and chin and hence seek for treatment. Others show more deformed faces due to inherited syndromes, e.g. Crouzon-Syndrome, Apert-Syndrome etc. or as a result of congenital diseases without inheritance (e.g. hemifacial microsomia). Other malformations comprise acquired diseases during childhood or adolescence (e.g. reduced growth of a jaw after trauma to the temporomandibular joint, i.e. the jaw-joint).

All these categories of diseases result in facial asymmetry or disfigurement. In addition to the general impairment of their health patients with these diseases suffer a great deal from their facial deformities. Since the human face plays a key role in interpersonal relationships it is essential not only to cure the underlying disease but also to predict the post-surgical morphology and appearance of the face. It is obvious that this is a critical issue for patients with facial deformities. Moreover, cranio-maxillofacial surgery has to strive for reconstruction of a balanced face. Even very subtle malformations of facial proportions can strongly affect the appearance of a face and determine on aesthetic aspects such as individual beauty [10].

Therefore, surgeons often face the problem of predicting a fair facial surface before the actual surgery is carried out. Figure 1 illustrates a typical malformation of a female's face and its correction by surgery. Normally, the planning of a maxillofacial surgical procedure is done by means of lateral X-ray images thereby predicting the $2 \mathrm{D}$ appearance of the postsurgical profile. Lateral X-ray images illustrating the actual and postsurgical profiles are presented in figures $1 \mathrm{~b}$ and $1 \mathrm{~d}$ respectively. It is clear that both surgeons and their patients have a strong demand for a method which 


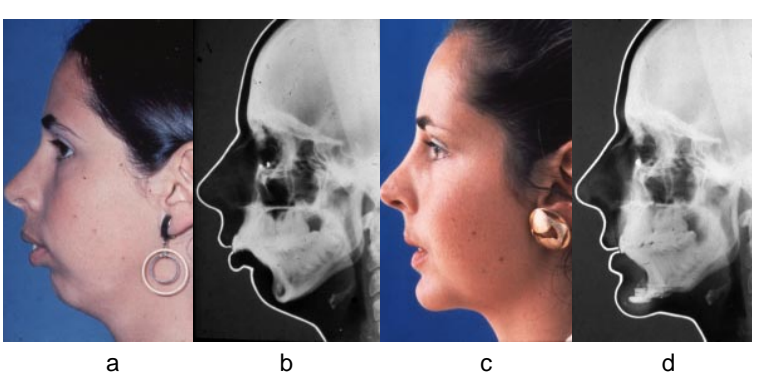

Figure 1: Example of a facial disharmony and its correction by maxillofacial surgery.

a) Presurgical facial shape contour (profile)

b) Presurgical lateral $X$-ray image

c) Postsurgical appearance after maxillofacial procedures

d) Postsurgical lateral X-ray image

enables them to compute highly realistic 3D pictures of the postsurgical shape. Any computation should be based on data available, or at least easy to obtain from the patient, e.g. CT or MRI scans in combination with laser scans of the surface. Obviously, the model has to capture the most important anatomical and mechanical parameters of the face in order to predict accurately the facial shape. Although various approaches to this problem can be found in literature, a satisfying solution does not exist yet. Contrary to existing approaches $[3,4,12,13,15]$, which mostly aim at real-time application, a face model suitable for our purpose has to base on more sophisticated Finite Element modeling, where true volumetric soft tissue models have to be combined with accurate geometric models of facial surface and individual skull.

\subsection{Previous Work}

Many approaches to facial simulation are based on surface models. Early works such as [19] restricted themselves to pure geometric deformations, mostly carried out directly on parametric surfaces. However, with physically based modeling paradigms, more realistic facial models arose, first based on mass spring systems and finite difference schemes (see e.g. [22]), later applying the Finite Element Method (FEM) for the solution of the inherent partial differential equations (see e.g. [5]). The common aspect of these models is the minimization of an energy functional of an elastic body subjected to deformation.

Koch et al. developed a surface based Finite Element approach which yielded very promising results both in the field of surgery simulation [15] and facial animation [14]. They approximated the volumetric behavior through a spring mesh connecting the thin plate spline surface to the skull. Lee et al. [17] presented a realistic model for facial animation which was based on prism elements combining the facial surface with a layered synthetic tissue model. Both, Koch and Lee, took into account anatomy and implemented an elaborate model of facial skin layers. Although both models have proved very powerful in the context of animation the lack of accurate volumetric physics diminishes their value in the field of surgery simulation.
Therefore, we focus our attention on volumetric models which implement as closely as possible the true behavior of tissue and skin. Keeve et al. introduced a computer-aided surgical planning system, first using a mass spring tissue model [12] and later incorporating linear prism elements [13]. Both approaches made use only of linear interpolation within the elements, and restricted themselves to linear elasticity in the implementation. The use of linear interpolation calls for a higher subdivision in order to reach the accuracy required in surgery simulation. This is a consequence of the fact, that convergence of Finite Element solutions can be achieved either by successive refinement of the mesh, or by increasing the polynomial degree of the interpolation on a fixed mesh. Furthermore, the use of prism elements restricts the geometry of the volume to shell-shaped structures.

In 1994, Morten Bro-Nielsen introduced the modeling of elastic solids using active cubes [3]. The approach combined snake-like feature extraction in CT data sets with energy minimization, but did not achieve the precision required for surgery simulation. In 1996, he presented a promising approach designed for real-time application, which modeled both linear elasticity and dynamic behavior using Finite Elements [4]. Aiming at real-time simulation, he again had to restrict himself to linear Finite Elements.

In the following paper we present a versatile framework for the Finite Element simulation of soft tissue for the application in facial surgery simulation. Our major contribution is the incorporation of higher order polynomial interpolation functions using a Bernstein-Bézier formulation, on the one hand, and an extension towards incompressible and nonlinear material behavior, on the other hand. In contrast to prior approaches we strive for accuracy instead of speed, and therefore accept higher computational costs. We take into consideration aspects both of underlying physics and of Finite Element discretization, and give some insight into the implementation of a prototype system. In order to be as general as possible from a geometrical point of view, we use Finite Elements defined over tetrahedra. This enables us to use arbitrary tetrahedralizations such as those presented in [21].

The outline of the paper is as follows: For reasons of readability, Section 2 deals with different aspects of the underlying physics, such as linear vs. nonlinear elasticity and incompressibility as well as some adaptations for our application. Section 3 discusses the solution strategies, i.e. the Finite Element discretization and the Bernstein-Bézier approach, and gives an idea of the implementation of the system. Results on synthetic data and on the Visible Human Data Set [18] are given in Section 4.

\section{Underlying Physics}

The analysis of soft tissue requires the idealization of tissue into a form that can be solved, the formulation of the mathematical and physical model, and the interpretation of the results. In our approach to soft tissue simulation we think of tissue as being elastic and therefore base on the theory of static elastomechanics. As for all continuous-system mathematical models, the governing differential equations must hold throughout the domain of the system. 
In order to derive the governing differential equations, we follow the variational approach, which essentially calculates the total potential $\Pi$ of the system - the functional of the problem - and invokes the stationarity of $\Pi$, i. e. $\delta \Pi=0$, which is equivalent to finding the configuration of minimal potential energy. In elastomechanics the potential $\Pi$ can be regarded as the elastic energy of the body minus the work done by externally applied forces. In the following sections we will first discuss linear elasticity and then go on to incompressible materials and nonlinearity.

\subsection{Linear Elasticity}

We think of an elastic body as the domain $\Omega \in R^{3}$ in the fixed coordinate system $X, Y, Z$ (see figure 2). The surface of the body is partly supported on the area $S_{u}$ with prescribed displacements $\mathbf{u}{ }^{u}$. In addition, the body is sub-

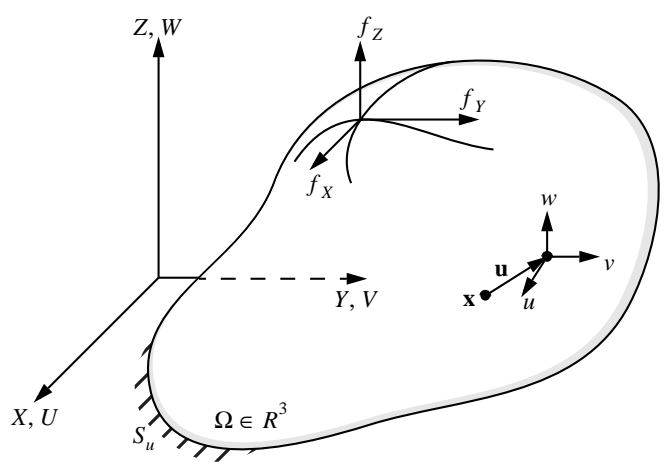

Figure 2: General three-dimensional elastic body

jected to externally applied forces $\mathbf{f}=\mathbf{f}\left(f_{X}, f_{Y}, f_{Z}\right)$. The forces together with the prescribed displacements yield a displacement field within the body which we denote by

$$
\mathbf{u}(X, Y, Z)=\left[\begin{array}{l}
u(X, Y, Z) \\
v(X, Y, Z) \\
w(X, Y, Z)
\end{array}\right]
$$

with $\mathbf{u}=\mathbf{u}^{S_{u}}$ on $S_{u}$. The displacement field itself yields corresponding strains $\varepsilon[1,23]$

$$
\varepsilon=\left[\begin{array}{llllll}
\varepsilon_{X X} & \varepsilon_{Y Y} & \varepsilon_{Z Z} & \gamma_{X Y} & \gamma_{Y Z} & \gamma_{Z X}
\end{array}\right]^{T}
$$

where

$$
\begin{gathered}
\varepsilon_{X X}=\frac{\partial u}{\partial X}, \quad \varepsilon_{Y Y}=\frac{\partial v}{\partial Y}, \quad \varepsilon_{Z Z}=\frac{\partial w}{\partial Z} \\
\gamma_{X Y}=\frac{\partial u}{\partial Y}+\frac{\partial v}{\partial X}, \quad \gamma_{Y Z}=\frac{\partial v}{\partial Z}+\frac{\partial w}{\partial Y}, \quad \gamma_{Z X}=\frac{\partial w}{\partial X}+\frac{\partial u}{\partial Z}
\end{gathered}
$$

with $\varepsilon_{X X}, \varepsilon_{Y Y}$, and $\varepsilon_{Z Z}$ as volumetric strain components and $\gamma_{X Y}, \gamma_{Y Z}$, and $\gamma_{Z X}$ as deviatoric or shear strain components.
The state of stress $\tau$ at an arbitrary point within $\Omega$ can be described as

$$
\tau=\left[\tau_{X X} \tau_{Y Y} \tau_{Z Z} \tau_{X Y} \tau_{Y Z} \tau_{Z X}\right]^{T} .
$$

In analogy to the strain components, $\tau_{X X}, \tau_{Y Y}$, and $\tau_{Z Z}$ describe the normal stresses in the coordinate directions whereas $\tau_{X Y}, \tau_{Y Z}$, and $\tau_{Z X}$ denote shear stresses. Assuming linear behavior we find for isotropic bodies the following linear relationship between stress and strain:

$$
\tau=\mathbf{C} \varepsilon .
$$

In (5), often referred to as the constitutive relation between stress and strain, $\mathbf{C}$ denotes the stress-strain material matrix

$$
\mathbf{C}=\frac{E}{(1+v)(1-2 v)}\left[\begin{array}{cccccc}
1-v & v & v & & \\
v & 1-v & v & 0 \\
v & v & 1-v & & \\
& & & \frac{1}{2}-v & \\
& 0 & & \frac{1}{2}-v & \\
& & & & & \frac{1}{2}-v
\end{array}\right]
$$

which can be derived using the generalized Hooke's law [20, 23]. E and $v$ denote Young's modulus and Poisson's ratio respectively. The magnitude of $E$ controls the stiffness of the material whereas $0 \leq v \leq 0.5$ measures its incompressibility. For $v=0.5$ we obtain total incompressibility which obviously cannot be handled by (6). The corresponding reformulation of the problem will be discussed in Section 2.2.

In essence, the problem can be formulated as follows: Given the geometry of the body, the loads $\mathbf{f}$, the boundary or support conditions $\mathbf{u}^{S_{u}}$, and the stress-strain relation of the material, calculate the displacement field $\mathbf{u}(X, Y, Z)$ within the body.

As mentioned above, the solution to the problem is the configuration $\mathbf{u}$ which minimizes the potential energy of the system. For linearly elastic materials we obtain the potential $\Pi$ as the difference of the elastic energy within the body and the work done by externally applied forces:

$$
\Pi=\frac{1}{2} \int_{V} \varepsilon^{T} \tau d V-\int_{V} \mathbf{u}^{T} \mathbf{f} d V .
$$

The principle of virtual work, or in terms of the Finite Element Method the principle of virtual displacements, states that the equilibrium of the body requires the equivalence of the total internal and external virtual work done by any imposed small virtual displacement satisfying the boundary conditions:

$$
\int_{V} \bar{\varepsilon}^{T} \tau d V=\int_{V} \overline{\mathbf{u}}^{T} \mathbf{f} d V
$$

The overbars on $\mathbf{u}$ and $\varepsilon$ denote virtual displacements and corresponding virtual strains respectively.

The equilibrium equation (8) can be obtained by invoking the stationarity of (7) (for a proof see e.g. [1]). 


\subsection{Incompressibility}

It is reasonable to think of human tissue as being almost incompressible because of its high water content. For the analysis of such media the pure displacement-based approach described in the previous section is not sufficient. We therefore employ a so-called mixed formulation which is far more efficient and can be thought of as a special case of the $H u$-Washizu variational principle (see $[1,24]$ ).

As it is difficult to predict accurately the pressure in the analysis of incompressible media, the key idea of the mixed formulation is the introduction of pressure as an additional variable. Assuming almost incompressible behavior, we can think of the volumetric strains being small compared to the deviatoric strains, and therefore reformulate the constitutive relation using indicial notation in the form

$$
\tau_{i j}=\kappa \varepsilon_{V} \delta_{i j}+2 G \varepsilon^{\prime}{ }_{i j}
$$

where $\delta_{i j}$ denotes the Kronecker delta, $\kappa$ is the bulk modulus

$$
\kappa=\frac{E}{3(1-2 v)}
$$

and $G$ is the shear modulus

$$
G=\frac{E}{2(1+v)}
$$

We thereby separate the volumetric strain $\varepsilon_{V}$,

$$
\varepsilon_{V}=\varepsilon_{k k}=\varepsilon_{X X}+\varepsilon_{Y Y}+\varepsilon_{Z Z} \approx \frac{\Delta V}{V},
$$

and the deviatoric strain components $\varepsilon_{i j}^{\prime}$,

$$
\varepsilon_{i j}^{\prime}=\varepsilon_{i j}-\frac{\varepsilon_{V}}{3} \delta_{i j}
$$

For the pressure in the body we have

$$
p=-\kappa \varepsilon_{V}
$$

where

$$
p=-\frac{\tau_{k k}}{3}=-\frac{\tau_{X X}+\tau_{Y Y}+\tau_{Z Z}}{3} .
$$

By gradually increasing $\kappa$ (which means that the Poisson ratio $v$ approaches 0.5$)$ the volumetric strain drops to zero. Using (9) and (14), the stress components become

$$
\tau_{i j}=-p \delta_{i j}+2 G \varepsilon_{i j}^{\prime} .
$$

For totally incompressible media, the displacement boundary conditions $\mathbf{u}$ must be compatible with the zero volumetric strain throughout the body. Further, in order to compute a unique solution, the pressure must be defined at some point in the body.
We consequently have to work with the unknown displacements $\mathbf{u}$ and the pressure $p$ as solution variables when using a mixed formulation. The principle of virtual work converts to

$$
\int_{V} \bar{\varepsilon}^{T} \tau^{\prime} d V-\int_{V} \bar{\varepsilon}_{V} p d V=\int_{V} \overline{\mathbf{u}}^{T} \mathbf{f} d V
$$

with the deviatoric stress vector $\tau^{\prime}$,

$$
\tau^{\prime}=\tau+p \delta,
$$

and the deviatoric strain vector $\varepsilon^{\prime}$,

$$
\varepsilon^{\prime}=\varepsilon-\frac{1}{3} \varepsilon_{V} \delta
$$

where $\delta$ is a vector of the Kronecker delta symbol.

In (17) we have separated the volumetric and deviatoric strain energies. The connection between the independent variables $p$ and $\mathbf{u}$ is provided by (14) written in integral form

$$
\int_{V}\left(\frac{p}{\kappa}+\varepsilon_{V}\right) \bar{p} d V=0
$$

\subsection{Nonlinear Extensions}

The above-mentioned procedures apply only to linear problems where the response $\mathbf{u}$ is a linear function of the applied loads. This is a consequence of the following three assumptions:

\section{- Small displacements/small strains}

The displacements $\mathbf{u}$ and the resulting strains must be small because all integrations are performed over the original volume. Further, in (12), we linearly approximated the volumetric strain which, in fact, is a nonlinear expression of type

$$
\varepsilon_{V}=\varepsilon_{X X}+\varepsilon_{Y Y}+\varepsilon_{Z Z}+\overbrace{\varepsilon_{X X} \varepsilon_{Y Y}+\varepsilon_{Y Y} \varepsilon_{Z Z}+\varepsilon_{Z Z} \varepsilon_{X X}+\varepsilon_{X X} \varepsilon_{Y Y} \varepsilon_{Z Z}}^{\text {nonlinear terms }}=\frac{\Delta V}{V}
$$

- Linearly elastic material

The constitutive relations derived above only reflect a linear relationship between stress and strain.

\section{- Boundary conditions remain unchanged}

In order to solve problems where at least one of these conditions is not met we have to elaborate on nonlinear schemes, which are still subject to extensive research activities in applied mechanics and material science.

Biomechanical studies have shown a highly nonlinear elastic response and hardening effects of facial tissue [2, 11]. We restrict ourselves to the static analysis of a nonlinear stress-strain relationship, still assuming small displacements and small strains (see figure 3). This enables us to go on working with the engineering strains defined by (2) and (3) instead of using total or updated Lagrangian formulations together with appropriate strain measures and the corresponding stress tensors (for a survey see [1]).

In order to derive the formulation of incremental nonlinear analysis we restate the principle of virtual work (8). Using indicial notation and writing virtual displacements 


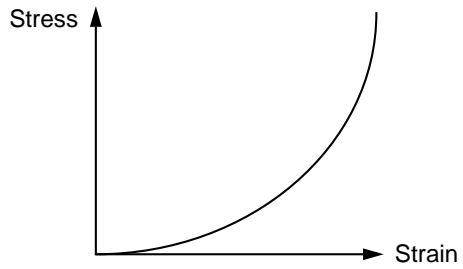

Figure 3: Nonlinear stress-strain relationship

and corresponding strains as variations $\delta u_{i}$ and $\delta \varepsilon_{i j}$, we have for the equilibrium condition of the body at time $t+\Delta t$

$$
\int_{V}{ }^{t+\Delta t} \tau_{i j} \delta \varepsilon_{i j} d V=\int_{V}{ }^{t+\Delta t} f_{i} \delta u_{i} d V
$$

In a static time independent analysis, the step-by-step incremental solution reduces to a one-step analysis if the total load is applied at once. However, for computational reasons, the analysis of such problems frequently requires an incremental solution where the variable $t$ denotes a number of loading steps to reach the total applied load.

As

$$
{ }^{t+\Delta t} \tau_{i j}={ }^{t+\Delta t} C_{i j r s}{ }^{t+\Delta t} \varepsilon_{r s}
$$

we are left with the problem of defining the entries of the stress-strain material matrix $\mathbf{C},{ }^{t+\Delta t} C_{i j r s}$. In order to achieve the desired nonlinear behavior of increasing stiffness with increasing strain we state Young's modulus as a function of the strain:

$$
E=E_{0} e^{A \varepsilon^{2}}
$$

with $E_{0}$ denoting Young's modulus for the relaxed material and $A$ serving as a measure of nonlinearity. For vanishing strains and for $A=0,(23)$ is equivalent to the linear case.

Note, that (23) relates to $1 \mathrm{D}$ problems, that is, we need a scalar $\varepsilon$ representing the actual strain. To this aim we define $\varepsilon$ in (23) either using the Frobenius norm $\varepsilon_{F}$ of the stress tensor or the arithmetic mean $\varepsilon_{A}$ of the magnitude of the tensor entries:

$$
\varepsilon_{F}=\sqrt{\sum_{i, j} \varepsilon_{i j}^{2}}, \quad \varepsilon_{A}=\frac{1}{9} \sum_{i, j}\left|\varepsilon_{i j}\right| \quad \text { with } \quad \varepsilon=\left[\begin{array}{ccc}
\varepsilon_{X X} & \gamma_{X Y} & \gamma_{Z X} \\
\gamma_{X Y} & \varepsilon_{Y Y} & \gamma_{Y Z} \\
\gamma_{Z X} & \gamma_{Y Z} & \varepsilon_{Z Z}
\end{array}\right]
$$

Note furthermore, that volumetric formulations of (23) are extremely difficult and open research issues [2].

Due to its nonlinearity, (21) will have to be solved approximately by referring all variables to a previously calculated known equilibrium configuration and subsequent linearization of the resulting equation. From (21), this results in

$$
\int_{V}{ }^{t} C_{i j r s} \varepsilon_{r s} \delta \varepsilon_{i j} d V=\int_{V}{ }^{t+\Delta t} f_{i} \delta u_{i} d V-\int_{V}{ }^{t} \tau_{i j} \delta \varepsilon_{i j} d V
$$

The integral term $\int_{V}{ }^{t} \tau_{i j} \delta \varepsilon_{i j} d V$ denotes the internal virtual work which results from the actual physical stresses ${ }^{t} \tau_{i j}$ at time $t$. Assuming that the approximate displacements and corresponding strains and stresses at time $t+\Delta t$ have been calculated according to (25), we can now define the error due to linearization as the out-of-balance virtual work,

$$
\text { Error }=\int_{V}{ }^{t+\Delta t} f_{i} \delta u_{i} d V-\int_{V}{ }^{t+\Delta t} \tau_{i j} \delta \varepsilon_{i j} d V .
$$

Therefore, in general, an iteration of (25) will be necessary to minimize the error (26).

\section{Solution Strategies}

In order to solve the above equations efficiently we employ the Finite Element method. In this section we describe the discretization process and discuss the shape functions we use. A short glimpse at the implementation concludes the section.

\subsection{Finite Element Discretization}

The discretization in FEM consists of two main aspects: Firstly, the domain of interest is subdivided into a finite and disjoint set of primitives, so-called Finite Elements, and secondly, we expand the solution within an element $m$ as a weighted sum of $n$ basis or shape functions $h_{i}$. This process is known as Galerkin projection:

$$
\tilde{\mathbf{u}}^{(m)}(x, y, z)=\sum_{i=0}^{n-1} \hat{u}_{i} h_{i}(x, y, z) .
$$

We can therefore state the displacement field within the element $m$ as

$$
\mathbf{u}^{(m)}(x, y, z)=\mathbf{H}^{(m)}(x, y, z) \hat{\mathbf{u}}^{(m)}
$$

where $\mathbf{H}^{(m)}$ denotes the displacement interpolation matrix and $\hat{\mathbf{u}}^{(m)}$ contains the $3 n$ nodal weights to the basis functions. The three spatial components of the displacement field are interpolated according to the structure of $\mathbf{H}^{(m)}$ and $\hat{\mathbf{u}}^{(m)}$ :

$$
\begin{aligned}
\mathbf{H}^{(m)} & =\left[\begin{array}{ccc}
h_{0} \ldots h_{n-1} & & 0 \\
& h_{0} \ldots h_{n-1} & \\
0 & & h_{0} \ldots h_{n-1}
\end{array}\right], \\
\hat{\mathbf{u}}^{(m)} & =\left[\begin{array}{llll}
\hat{u}_{x_{0}} \ldots \hat{u}_{x_{n-1}} & \hat{u}_{y_{0}} \ldots \hat{u}_{y_{n-1}} & \hat{u}_{z_{0}} \ldots \hat{u}_{z_{n-1}}
\end{array}\right]^{T} .
\end{aligned}
$$

Consequently, we have for the interpolation of the element strains

$$
\varepsilon^{(m)}(x, y, z)=\mathbf{B}^{(m)}(x, y, z) \hat{\mathbf{u}}^{(m)}
$$


with the strain interpolation matrix $\mathbf{B}^{(m)}$ corresponding to (3)

$$
\mathbf{B}^{(m)}=\left[\begin{array}{ccc}
\frac{\partial}{\partial x}\left[h_{0} \ldots h_{n-1}\right] & \mathbf{0} & \mathbf{0} \\
\mathbf{0} & \frac{\partial}{\partial y}\left[h_{0} \ldots h_{n-1}\right] & \mathbf{0} \\
\mathbf{0} & \mathbf{0} & \frac{\partial}{\partial z}\left[h_{0} \ldots h_{n-1}\right] \\
\frac{\partial}{\partial y}\left[h_{0} \ldots h_{n-1}\right] & \frac{\partial}{\partial x}\left[h_{0} \ldots h_{n-1}\right] & \mathbf{0} \\
\mathbf{0} & \frac{\partial}{\partial z}\left[h_{0} \ldots h_{n-1}\right] \frac{\partial}{\partial y}\left[h_{0} \ldots h_{n-1}\right] \\
\frac{\partial}{\partial z}\left[h_{0} \ldots h_{n-1}\right] & \mathbf{0} & \frac{\partial}{\partial x}\left[h_{0} \ldots h_{n-1}\right]
\end{array}\right] .
$$

Note specifically that $\mathbf{B}^{(m)}$ contains first order derivatives of the shape functions.

Using (28) and (30) for the interpolation of the virtual displacement and virtual strains respectively, we can rewrite the principle of virtual displacements (8) as

$$
\overline{\hat{\mathbf{U}}}^{T}\left[\sum_{m} \int_{V^{(m)}} \mathbf{B}^{(m) T} \mathbf{C}^{(m)} \mathbf{B}^{(m)} d V^{(m)}\right] \hat{\mathbf{U}}=\overline{\hat{\mathbf{U}}}^{T}\left[\sum_{m} \int_{V^{(m)}} \mathbf{H}^{(m) T} \mathbf{f}^{(m)} d V^{(m)}\right](32)
$$

where $\hat{\mathbf{U}}$ and $\overline{\hat{\mathbf{U}}}$ are vectors containing all $3 N$ nodal weights of an element assemblage with a total of $N$ nodes and $\mathbf{H}^{(m)}, \mathbf{B}^{(m)}$, and $\mathbf{C}^{(m)}$ are matrices of corresponding size which are zero everywhere except at the places which correspond to the weights of element $m$.

Applying the principle of virtual displacement $3 N$ times using unit virtual displacements for all components of $\overline{\hat{\mathbf{U}}}$ yields the governing system of linear equations

$$
\mathbf{K} \mathbf{U}=\mathbf{R},
$$

where $\mathbf{U} \equiv \hat{\mathbf{U}}$. In (33), $\mathbf{K}$ denotes the symmetric positivedefinite global stiffness matrix

$$
\mathbf{K}=\sum_{m} \int_{V^{(m)}} \mathbf{B}^{(m) T} \mathbf{C}^{(m)} \mathbf{B}^{(m)} d V^{(m)},
$$

$\mathbf{R}$ the global force vector

$$
\mathbf{R}=\sum_{m} \int_{V^{(m)}} \mathbf{H}^{(m) T} \mathbf{f}^{(m)} d V^{(m)}
$$

and $\mathbf{U}$ the unknown displacement vector. The summation signs in (34) and (35) can be regarded as the so-called assembly of the local stiffness matrices $\int_{V^{(m)}} \mathbf{B}^{(m) T} \mathbf{C}^{(m)} \mathbf{B}^{(m)} d V^{(m)}$ and the local force vectors $\int_{V^{(m)}}^{V^{(m)}} \mathbf{H}^{(m) T} \mathbf{f}^{(m)} d V^{(m)}$ into one global matrix and vector respectively.

The interpolation of the displacements remains the same in the case of incompressibility, whereas the strain has to be separated into volumetric and deviatoric part

$$
\begin{aligned}
& \varepsilon_{V}^{(m)}(x, y, z)=\mathbf{B}_{V}^{(m)}(x, y, z) \hat{\mathbf{u}}^{(m)} \\
& \varepsilon^{(m)}(x, y, z)=\mathbf{B}_{D}^{(m)}(x, y, z) \hat{\mathbf{u}}^{(m)}
\end{aligned}
$$

and the pressure is interpolated according to

$$
p^{(m)}(x, y, z)=\mathbf{H}_{p}^{(m)}(x, y, z) \hat{\mathbf{p}}^{(m)}
$$

where $\mathbf{H}_{p}^{(m)}$ denotes the pressure interpolation matrix

$$
\mathbf{H}_{p}^{(m)}=\left[h_{p 0} \ldots h_{p k}\right] .
$$

As can be seen from (38), the basis functions for the interpolation of pressure do not necessarily have to be the same as for the interpolation of the displacements.

The structure of the resulting system of linear equations consequently becomes

$$
\left[\begin{array}{ll}
\mathbf{K}_{u u} & \mathbf{K}_{u p} \\
\mathbf{K}_{p u} & \mathbf{K}_{p p}
\end{array}\right]\left[\begin{array}{l}
\hat{\mathbf{u}} \\
\hat{\mathbf{p}}
\end{array}\right]=\left[\begin{array}{l}
\mathbf{R} \\
\mathbf{0}
\end{array}\right]
$$

with the partial stiffness matrices

$$
\begin{aligned}
& \mathbf{K}_{u u}=\sum_{m} \int_{V^{(m)}} \mathbf{B}_{D}^{(m) T} \mathbf{C}^{(m)} \mathbf{B}_{D}^{(m)} d V^{(m)} \\
& \mathbf{K}_{u p}=\mathbf{K}_{p u}^{T}=-\sum_{m} \int_{V^{(m)}} \mathbf{B}_{V}^{(m) T} \mathbf{H}_{p}^{(m)} d V^{(m)} \\
& \mathbf{K}_{p p}=-\sum_{m} \int_{V^{(m)}} \mathbf{H}_{p}^{(m) T} \frac{1}{\kappa} \mathbf{H}_{p}^{(m)} d V^{(m)}
\end{aligned}
$$

and the corresponding interpolation matrices

$$
\begin{aligned}
& \mathbf{B}_{V}^{(m)}=\left[\begin{array}{llllll}
1 & 1 & 1 & 0 & 0 & 0
\end{array}\right] \mathbf{B}^{(m)} \\
& \mathbf{B}_{D}^{(m)}=\frac{1}{3}\left[\begin{array}{ccccc}
2 & -1 & -1 & & \\
-1 & 2 & -1 & & 0 \\
-1 & -1 & 2 & & \\
& & 3 & \\
0 & & 3 & \\
& & & & 3
\end{array}\right] \mathbf{B}^{(m)} \text {. }
\end{aligned}
$$

The partial stiffness matrices (40) can be found by substituting (28), (36), and (37) into (17) and (20). The deviatoric stress-strain relational matrix $\mathbf{C}^{\prime}(m)$ follows from (16) to

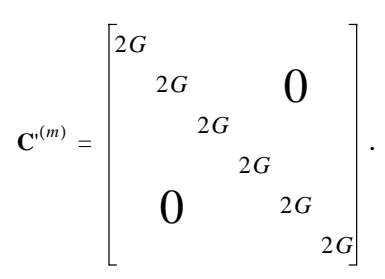

For the incremental step-by-step solution in the nonlinear case we are left with the problem of finding the state of equilibrium of a body at time $t+\Delta t$ assuming a known configuration at time $t$. The equilibrium at time $t$ is equivalent to a vanishing out-of-balance virtual work in (26) and can be expressed as

$$
{ }^{t+\Delta t} \mathbf{R}-{ }^{t+\Delta t} \mathbf{F}=\mathbf{0}
$$


where the vector ${ }^{t+\Delta t} \mathbf{R}$ lists the externally applied forces at time $t+\Delta t$

$$
{ }^{t+\Delta t} \mathbf{R}=\sum_{m} \int_{V^{(m)}} \mathbf{H}^{(m) T}{ }^{t+\Delta t} \mathbf{f}^{(m)} d V^{(m)}
$$

and the stress load vector ${ }^{t+\Delta t} \mathbf{F}$ lists the nodal point forces that correspond to the element stresses at time $t+\Delta t$,

$$
{ }^{t+\Delta t} \mathbf{F}=\sum_{m} \int_{V^{(m)}} \mathbf{B}^{(m) T t+\Delta t} \tau^{(m)} d V^{(m)} .
$$

Since the solution at time $t$ is known, we can calculate the increment to the solution after a suitably small time increment $\Delta t$ using

$$
{ }^{t+\Delta t} \mathbf{F}={ }^{t} \mathbf{F}+\mathbf{F}
$$

where $\mathbf{F}$ is the increment to the stress load vector corresponding to the increment in element displacements and stresses from time $t$ to time $t+\Delta t$. This vector can be approximated using a tangent stiffness matrix ${ }^{t} \mathbf{K}$ which corresponds to the material conditions at time $t$,

$$
\mathbf{F} \approx{ }^{t} \mathbf{K} \mathbf{U}
$$

where $\mathbf{U}$ is an incremental vector to the displacements at time $t$ and

$$
{ }^{t} \mathbf{K}=\frac{\partial^{t} \mathbf{F}}{\partial^{t} \mathbf{U}}
$$

Substituting (47) and (46) into (43) yields the matrix equation corresponding to (25)

$$
{ }^{t} \mathbf{K} \mathbf{U}={ }^{t+\Delta t} \mathbf{R}-{ }^{t} \mathbf{F},
$$

and by solving for $\mathbf{U}$ we can calculate an approximation to the displacements at time $t+\Delta t$,

$$
{ }^{t+\Delta t} \mathbf{U} \approx{ }^{t} \mathbf{U}+\mathbf{U} .
$$

Due to the approximation in (47), we cannot use the result of (50) for the computation of the strains and stresses at time $t+\Delta t$ and proceed to the next time step. Depending on the size of time steps, such a solution might be very unstable and lead to significant errors. We therefore have to iterate the solution of (49) until the condition (43) is met to a sufficiently accurate extent.

The widely used iteration methods in Finite Element analysis are based on the classical Newton-Raphson technique [1]. That is, having calculated an increment to the total displacement vector $\mathbf{U}$, we can use this vector instead of the vector known from time $t$ to calculate the stress load vector and the tangent stiffness matrix. This results in the following nested iteration, for $i=1,2,3, \ldots$,

$$
\begin{aligned}
& { }^{t+\Delta t} \mathbf{K}^{(i-1)} \Delta \mathbf{U}^{(i)}={ }^{t+\Delta t} \mathbf{R}-{ }^{t+\Delta t} \mathbf{F}^{(i-1)} \\
& { }^{t+\Delta t} \mathbf{U}^{(i)}={ }^{t+\Delta t} \mathbf{U}^{(i-1)}+\Delta \mathbf{U}^{(i)} \\
& { }^{t+\Delta t} \mathbf{F}^{(i)}={ }^{t+\Delta t} \mathbf{K}^{(i)}{ }^{t+\Delta t} \mathbf{U}^{(i)}
\end{aligned}
$$

with the initial conditions

$$
{ }^{t+\Delta t} \mathbf{U}^{(0)}={ }^{t} \mathbf{U}, \quad{ }^{t+\Delta t} \mathbf{K}^{(0)}={ }^{t} \mathbf{K}, \quad{ }^{t+\Delta t} \mathbf{F}^{(0)}={ }^{t} \mathbf{F} .
$$

The iteration of (51) is continued until the out-of-balance load vector ${ }^{t+\Delta t} \mathbf{R}-{ }^{t+\Delta t} \mathbf{F}^{(i-1)}$ gets very small and therefore the increment in the nodal point displacements $\Delta \mathbf{U}^{(i)}$ meets a convergence criterion as for instance

$$
\frac{\left\|\Delta \mathbf{U}^{(i)}\right\|_{2}}{\| t+\Delta t} \mathbf{U}^{(i)} \|_{2} \leq \varepsilon .
$$

As it is very expensive to evaluate the tangent matrix ${ }^{t+\Delta t} \mathbf{K}^{(i-1)}$ at every step of the iteration, it may be more efficient to evaluate a new tangent stiffness matrix only at the beginning of each load step (modified Newton-Raphson iteration).

\subsection{Bernstein-Bézier Shape Functions}

So far, we have not made any assumptions about the kind of elements used in the discretization. In this section we will introduce the notion of tetrahedral Bernstein-Bézier elements which means that our interpolation scheme employs Bernstein polynomials defined over the elements of an irregular tetrahedralization.

Bernstein polynomials are defined over local barycentric coordinates $\left(L_{1}, L_{2}, L_{3}, L_{4}\right)$. The interpolation of the element geometry is of the form

$$
x=\sum_{i=1}^{q} h_{i} x_{i}, \quad y=\sum_{i=1}^{q} h_{i} y_{i}, \quad z=\sum_{i=1}^{q} h_{i} z_{i}
$$

with $h_{i}=f\left(L_{1}, L_{2}, L_{3}, L_{4}\right)$. We employ a subparametric formulation using a linear interpolation of the geometry because of the assumption of small displacements and the linear tetrahedralization. Every point within the tetrahedron is therefore defined by

$$
\mathbf{P}\left(L_{1}, L_{2}, L_{3}, L_{4}\right)=\mathbf{P}_{1} L_{1}+\mathbf{P}_{2} L_{2}+\mathbf{P}_{3} L_{3}+\mathbf{P}_{4} L_{4}
$$

with $L_{1}+L_{2}+L_{3}+L_{4} \equiv 1$ and $\mathbf{P}_{i}$ denoting the corner vertices of the tetrahedron.

In order to evaluate the strain interpolation matrix (31) we need derivatives of element displacements with respect to the global coordinates. The relation of $X, Y, Z$ to $L_{1}, L_{2}, L_{3}, L_{4}$ derivatives is given by the Jacobian as

$$
\left[\begin{array}{l}
\frac{\partial}{\partial X} \\
\frac{\partial}{\partial Y} \\
\frac{\partial}{\partial Z}
\end{array}\right]=\mathbf{J}^{-1}\left[\begin{array}{l}
\frac{\partial}{\partial L_{1}} \\
\frac{\partial}{\partial L_{2}} \\
\frac{\partial}{\partial L_{3}}
\end{array}\right] \text { with } \mathbf{J}=\left[\begin{array}{l}
\frac{\partial X}{\partial L_{1}} \frac{\partial Y}{\partial L_{1}} \frac{\partial Z}{\partial L_{1}} \\
\frac{\partial X}{\partial L_{2}} \frac{\partial Y}{\partial L_{2}} \\
\frac{\partial Z}{\partial L_{2}} \\
\frac{\partial X}{\partial L_{3}} \frac{\partial Y}{\partial L_{3}} \frac{\partial Z}{\partial L_{3}}
\end{array}\right] .
$$

The Jacobian and its inverse are constant because of the linear interpolation (55). 
Using Bernstein polynomials, the weights of the Galerkin projection we are solving for can be regarded as Bézier control vertices. Barycentric tetrahedral Bernstein polynomials of degree $n$ are of the form [8]

$$
B_{i j k l}^{n}\left(L_{1}, L_{2}, L_{3}, L_{4}\right)=\left(\begin{array}{c}
n \\
i, j, k, l
\end{array}\right) L_{1}{ }^{i} L_{2}{ }^{j} L_{3}{ }^{k} L_{4}{ }^{l}=\frac{n !}{i ! j ! k ! ! !} L_{1}{ }^{i} L_{2}{ }^{j} L_{3}{ }^{k} L_{4}{ }^{l} .
$$

The corresponding Bézier patch is defined by

$$
\mathbf{b}\left(L_{1}, L_{2}, L_{3}, L_{4}\right)=\sum_{i, j, k, l \geq 0}^{i+j+k+l=n} \mathbf{b}_{i j k l} B_{i j k l}^{n}\left(L_{1}, L_{2}, L_{3}, L_{4}\right)
$$

where $\mathbf{b}_{i j k l}$ denote the control vertices of the patch. Figure 4 depicts the control nets of linear, quadratic, and cubic tetrahedral Bézier patches and three triangular cubic Bernstein polynomials.
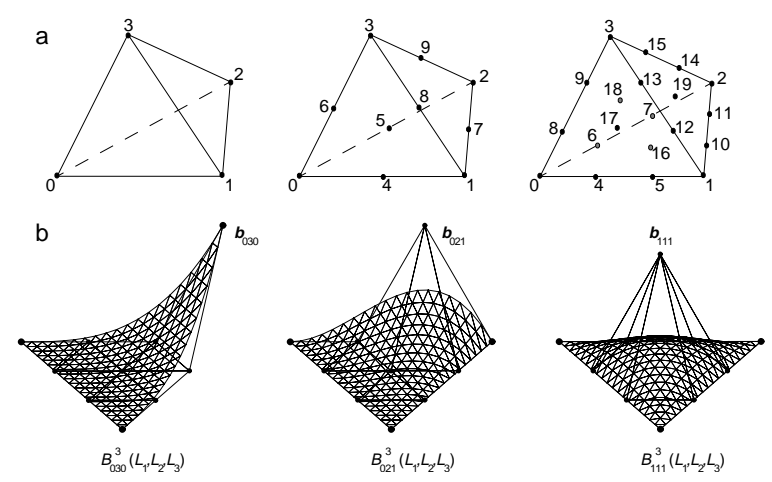

Figure 4: a) Control nets of linear, quadratic, and cubic Bézier patches, b) Cubic triangular Bernstein polynomials

In summary, the use of Bernstein-Bézier based FEM has the following advantages:

- Suitability for geometric modeling

For years, Bézier surfaces and volumes have proved their suitability for CAGD [9, 8]. Their formulation is well-studied and the inherent endpoint interpolation lends itself well to Finite Element modeling.

- Integral polynomial form of arbitrary degree

- Analytical derivatives and integrals

For integral polynomial functions $f$, the integral over the tetrahedral domain $V^{(m)}$

$$
\int_{V^{(m)}} f\left(L_{1}, L_{2}, L_{3}, L_{4}\right) d V^{(m)}=\int_{0}^{1} \int_{0}^{\left(1-L_{1}\right)\left(1-L_{1}-L_{2}\right)} \int_{0}^{f} f|\mathbf{J}| d L_{3} d L_{2} d L_{1}
$$

can be evaluated analytically using a closed formula. This considerably speeds up the computation of the local stiffness matrices and force vectors.

- Fast subdivision

The well-known De Casteljau algorithm [9] can be used both for the visualization of the resulting surface and for fast subdivision and refinement schemes, such as a progressive refinement of the solution (see figure 5). The right superscript $n$ of the control vertices in figure 5 denotes the Bézier net after $n$ De Casteljau steps.

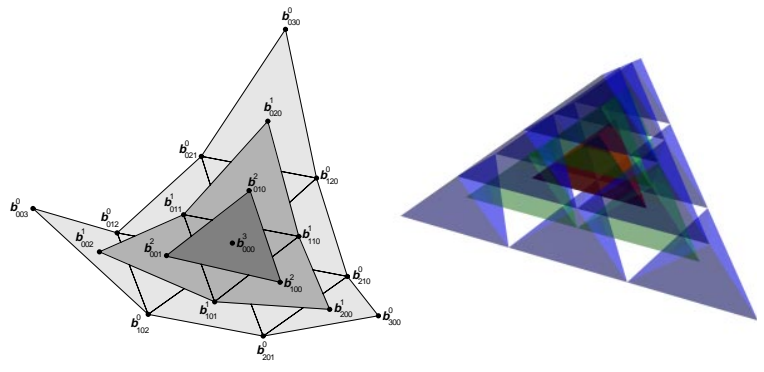

Figure 5: Visualization of $2 D$ and $3 D$ de Casteljau Algorithm for cubic patches

\subsection{Implementation}

We have implemented a C++ prototype embedded in AVS/ Express, which allows to perform all the various types of computations outlined above. In Section 4 we will show results of some comparisons.

The calculation of the local stiffness matrices is done analytically when assembling the global matrix. The corresponding code was generated by Maple.

In order to solve the system of linear equations we either use a conjugate gradient solver with incomplete Cholesky preconditioning $[6,7]$ or an LU decomposition [16] for the indefinite matrices arising from totally incompressible materials.

\section{Results}

The implementation offers a great choice of possible simulation types. We can interpolate to a lower or higher degree, choose between compressible and incompressible media, and perform linear or nonlinear calculations. In order to evaluate the various aspects and parameters of the presented model we first experienced the system on a synthetic block of soft tissue. In addition, we simulated lower jaw bone surgery on the Visible Human Data Set [18].

\subsection{Experiencing the System}

As a first comparison we want to examine the effect of the degree of interpolation on the outcome of the simulation. To this aim, we regularly subdivide a block of elastic soft tissue into tetrahedra. This block is fixed at its lower corner nodes and exposed to a vertical force centered at the top of the block (see figure 6). We perform a simulation of linearly elastic compressible material for increasing forces using linear, quadratic, and cubic interpolation.

It is evident that increasing the degree of interpolation and thus incrementing the number of degrees of freedom, introduces more and more detail to the resulting shape. As a consequence, linear elements are incapable of bending, which leeds to a block-like outcome. Due to the linear setting the resulting displacement is proportional to the applied forces.

The next experiment deals with the effects of incompressible media. Again, we take an elastic block as described before and perform a linear, incompressible calculation for two different forces. The interpolation of dis- 


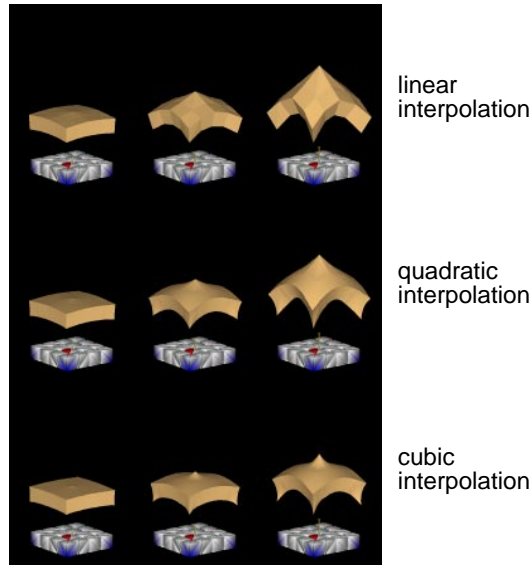

Figure 6: Comparison of linear, quadratic, and cubic Bézier elements; $E=1.0, v=0.25$, applied forces $0.1,0.5,1.0$

placements is quadratic whereas the pressure is interpolated linearly. It is a result of Finite Element theory, that in order to prevent an element from so-called locking, the interpolation of pressure in incompressible computations has to be of a lower degree than the interpolation of displacements [1].

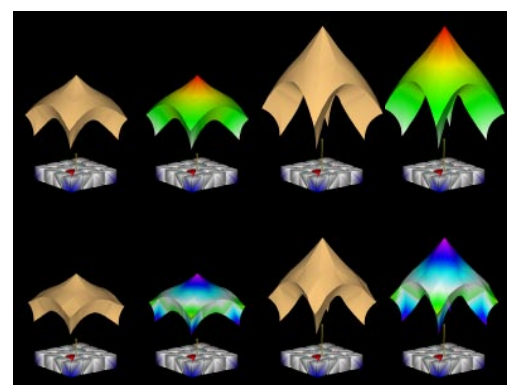

compressible analysis

ncompressible analysis

Figure 7: Comparison of compressible and incompressible analysis with quadratic elements; $E=1.0, v=0.5$, applied forces 1.0, 2.0

The results are shown in figure 7. It is interesting to see that the results are very similar. However, the incompressible material reveals to be somewhat stiffer and is harder to bend. The coloring in the second and fourth column visualizes the displacement and pressure in compressible and incompressible analysis respectively. Red stands for maximal displacement, magenta for minimal pressure.

The next experiment on synthetic data deals with the comparison between linear and nonlinear analysis. We expose an elastic block to increasing forces and examine the resulting maximal displacement for linear and nonlinear analysis (figure 8).

The results confirm our expectations: the linear element shows the expected linear relation between applied force and resulting displacement, whereas the stiffness of the nonlinear element increases with the strain.

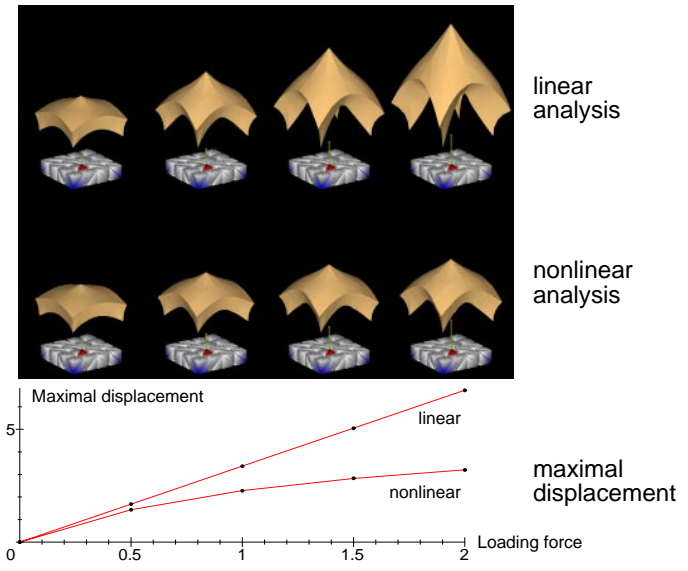

Figure 8: Comparison of linear and nonlinear analysis with quadratic elements; $E=1.0, v=0.25, A=5$, applied force $0.5,1.0,1.5,2.0$

\subsection{Responses of Human Facial Soft Tissue}

The last experiment presents first results of facial tissue simulation on the Visible Human Data Set. To simulate the advancement of the lower jaw, we prescribed the nodal displacements at the jaw bone on the inside of the tissue according to the desired move. This leads to a corresponding loading vector. In this case, we performed a compressible linear analysis because of the little effects of incompressible analysis and restricted ourselves to linear computation because of the small displacements resulting from the simulated surgical procedure.

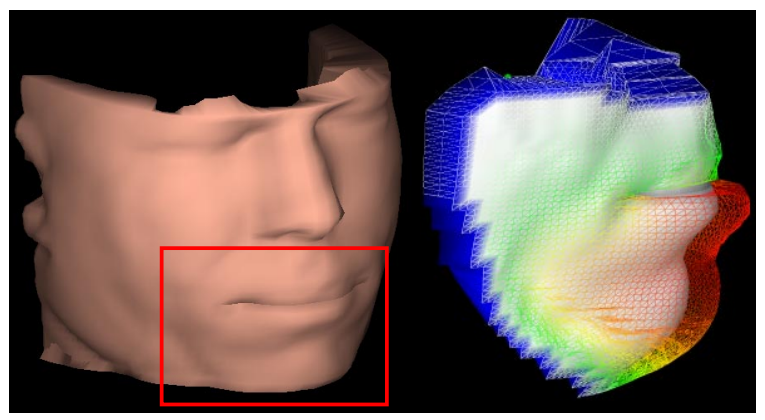

Figure 9: Surgical procedure on the lower jaw; linear elasticity, compressible analysis with quadratic elements, $E=1.0, v=0.25$. The loading forces are defined by prescribed displacements on the jaw bone.

(Data source: Visible Human Project, Courtesy National Library of Medicine)

Figure 9 shows the result of the simulation as a wireframe mesh superimposed on the original facial surface. In spite of the simplifying assumptions made to compute this model we obtain highly realistic displacement fields. 


\subsection{Performance}

The performance of the system heavily depends on the type of simulation used. A nonlinear analysis is computationally far more expensive than linear simulation because of the stepwise loading and the required iterations at each loading step. Since the Bernstein-Bézier elements make the computation of the stiffness matrix extremely fast, most of the simulation time is spent on solving the resulting system of equations. Hence, the speed of the different solvers and preconditioners strongly influences the total simulation time.

The simulation of the example shown in figure 9 took 44 minutes on a SGI Indigo ${ }^{2}$, R10000, 195 MHz, 512 MB. The part of the jaw was made of 4108 quadratic tetrahedra which resulted in a 17'904 x 17'904 stiffness matrix.

\section{Conclusions and Future Work}

We presented an implementation of several Finite Element models for soft tissue simulation. We explained some of the theoretical background of linear and nonlinear elasticity as well as the Finite Element discretization based on a Bernstein-Bézier formulation. Furthermore, we discussed the results on the basis of a synthetic example and a surgical procedure on the Visible Human Data Set.

The main drawback of the current approach is the missing global $\mathrm{C}^{1}$ continuity. The resulting surface is therefore of lower quality than in [15]. Moreover, the finite elements presented in this paper are incompatible, that is, the $\mathrm{C}^{0}$ continuous interpolation across element boundaries cannot capture the continuity required for cross-boundary derivatives. As a consequence, the development of a globally $\mathrm{C}^{1}$ continuous FE model is subject to current and future research.

In addition, we aim at the development of a prototype for medical applications followed by a case study with individual patients. It will enable us to compare the results of different Finite Element models with those achieved by actual surgical procedures. Only then will it be possible to finally choose the most appropriate type of simulation.

\section{Acknowledgement}

The authors would like to thank Christoph Zehnder for implementing parts of the system.

\section{References}

[1] K.-J. Bathe. Finite element procedures. Prentice Hall, Englewood Cliffs, 2nd edition, 1996. Rev. of: Finite element procedures in engineering analysis. 1982.

[2] S. R. Bodner and M. B. Rubin. "A study of the extensional properties of excised facial skin and smas." In Fifth Pan American Congress of Applied Mechanics - PACAM V, 1997. January 2-4, 1997, San Juan, Puerto Rico.

[3] M. Bro-Nielsen. "Modelling elasticity in solids using active cubes - application to simulated operations." In Proc. Computer Vision, Virtual Reality, and Robotics in Medicine (CVRMed'95), 1995.

[4] M. Bro-Nielsen and S. Cotin. "Real-time volumetric deformable models for surgery simulation using finite elements and condensation." In Proc. EUROGRAPHICS'96, pages C57-C66, 1996.

[5] G. Celniker and D. Gossard. "Deformable curve and surface finite elements for free-form shape design." In T. W.
Sederberg, editor, Computer Graphics (SIGGRAPH '91 Proceedings), volume 25, pages 257-266, July 1991.

[6] J. Dongarra, A. Lumsdaine, R. Pozo, and K. Remington. "A sparse matrix library in c++ for high performance architectures." In Proceedings of the Second Object Oriented Numerics Conference, pages 214-218, 1994.

[7] J. Dongarra, A. Lumsdaine, R. Pozo, and K. Remington. $M V++$, IML++, SparseLib++ - Iterative methods for efficient sparse matrix computations. National Institute of Standards and Technology, 1996. http://math.nist.gov/ mcsd/Staff/RPozo/.

[8] G. Farin. "Triangular bernstein-bézier patches." Computer Aided Geometric Design, 3:83-127, 1986.

[9] G. Farin. Curves and Surfaces for Computer Aided Geometric Design. Academic Press, 1990.

[10] L. G. Farkas. Anthropometry of the Head and Face. Raven Press, 2nd edition, 1994.

[11] Y. C. Fung. Biomechanics: mechanical properties of living tissues. Springer, 2nd edition, 1993.

[12] E. Keeve, S. Girod, and B. Girod. "Computer-aided craniofacial surgery." In Proc. Computer Assisted Radiology CAR'96, pages 757-762, june 1996.

[13] E. Keeve, S. Girod, P. Pfeifle, and B. Girod. "Anatomybased facial tissue modeling using the finite element method.” In Proc. of IEEE Visualization'96, Nov. 1996.

[14] R. M. Koch, M. H. Gross, and A. A. Bosshard. "Emotion editing using finite elements." In Proc. EUROGRAPHICS'98, 1998 .

[15] R. M. Koch, M. H. Gross, F. R. Carls, D. F. von Büren, G. Fankhauser, and Y. I. H. Parish. "Simulating facial surgery using finite element models." In SIGGRAPH 96 Conference Proceedings, Annual Conference Series, pages 421-428. ACM SIGGRAPH, Addison Wesley, Aug. 1996. held in New Orleans, Louisiana, 4-9 August 1996.

[16] K. S. Kundert and A. Sangiovanni-Vincentelli. A Sparse Linear Equation Solver. Department of Electrical Engineering and Computer Sciences, University of California, Berkeley, 1988. http://veng-server.pharmacy.uiowa.edu/ CD_1/netlib/sparse/00_index.htm.

[17] Y. Lee, D. Terzopoulos, and K. Waters. "Realistic face modeling for animation." In R. Cook, editor, SIGGRAPH 95 Conference Proceedings, Annual Conference Series, pages 55-62. ACM SIGGRAPH, Addison Wesley, Aug. 1995. held in Los Angeles, California, 06-11 August 1995.

[18] National Library of Medicine. The Visible Human Project. http://www.nlm.nih.gov/extramural_research.dir/ visible_human.html, 1995.

[19] F. I. Parke. "Parameterized models for facial animation." IEEE Computer Graphics and Applications, 2:61-68, Nov. 1982.

[20] J. D. Renton. Applied Elasticity: Matrix and Tensor Analysis of Elastic Continua. Ellis Horwood series in mechanical engineering. Ellis Horwood Limited, 1987.

[21] O. G. Staadt, M. H. Gross, and R. Weber. "Multiresolution compression and reconstruction." In Proceedings of IEEE Visualization 1997, pages 337-346. IEEE, 1997.

[22] D. Terzopoulos, J. Platt, A. Barr, and K. Fleischer. "Elastically deformable models." In M. C. Stone, editor, Computer Graphics (SIGGRAPH '87 Proceedings), volume 21, pages 205-214, July 1987.

[23] S. Timoshenko and J. N. Goodier. Theory of Elasticity. McGraw-Hill, New York, 3rd edition, 1970.

[24] K. Washizu. Variational Methods in Elasticity and Plasticity. Pergamon Press, Elmsford, NY, 1975. 